\title{
Chronic osteomyelitis on an old compound fracture
}

\author{
A. Shrestha ${ }^{1}$, S. Kumar Sah ${ }^{1}$ \\ ${ }^{1}$ Intern (MBBS), College of Medical Sciences - Teaching Hospital, Bharatpur, Chitwan, Nepal.
}

\begin{abstract}
Osteomyelitis is a common complication of compound fracture of long bones which is difficult to treat. We present a similar case of chronic osteomyelitis on an old malunited compound fracture of distal third left femur in a nine years old child. Management of such cases requires surgery with immobilization, long term appropriate antibiotics and intensive follow up.
\end{abstract}

\section{Keywords: chronic osteomyelitis, compound fracture, surgery.}

\section{Introduction}

Infection is common in compound fracture and its management is difficult. It comprises a spectrum from acute suppuration of a fracture hematoma to indolent drainage from a chronic bone abscess. Osteomyelitis can be grouped into four categories as described by Cierny and Meder. ${ }^{1}$ Hematogenous or medullary osteomyelitis results from blood borne bacteria that reach the area of injury and may grow in fracture hematoma. Superficial osteomyelitis is a contiguous focus infection of bone that occurs when an exposed infected necrotic surface of bone lies at the base of a soft tissue wound. Localized osteomyelitis is a full thickness, cortical sequestration which can be removed surgically without compromising bony stability. It is commonly found as bone infection adjacent to an implant. Diffuse osteomyelitis occurs when the underlying fracture is not healed. It includes bone infections with a loss of bony stability and requires bone

Correspondence: A. Shrestha

E-mail: asis.shrth@gmail.com resection to arrest the disease process, followed by bony reconstruction. ${ }^{2}$

The principles of treatment of chronic post-traumatic osteomyelitis include a pus culture to identify the causative organism, and finding out the sensitive antibiotics which can be administered after surgery. Other important steps include debridement of the infected focus, tackling the resultant dead space, and controlling any fracture instability, besides increasing blood supply locally.

\section{Case Report}

A 9 year old girl presented to orthopedics outdoor department with history of pain on left thigh and discharge of pus from medial aspect of the left thigh. On inquiry, she gave a history of fall on playground 2 years back. She was diagnosed to have a compound fracture left femur for which wound debridement was done and a plaster cast was applied for 2 months in a 


\section{A. Shrestha et al. Chronic osteomyelitis on an old compound fracture}

local hospital. Three months later, she had a mild swelling, pus discharge on and off and pain over the left thigh on the fracture site with difficulty in walking. She was treated with antibiotics and dressings. Patient had no complaint for some months. She then presented to us with pus discharge from left thigh. She complained of mild pain over the thigh but no fever.

Local examination of left thigh showed a $2 \mathrm{~cm} X 2 \mathrm{~cm}$ crusted wound with a pus discharging sinus over the medial aspect. A scar of previous wound debridement was also visible on the same area. She had an antalgic gait. On palpation, tenderness was present. Range of motion of hip and knee was normal. There was shortening of one inch in left lower limb. Distal neurovascular status was intact. Blood counts and biochemical profile were within the normal range. Pus culture showed no organisms.

Anteroposterior and lateral view of plain radiograph of left thigh showed old malunited fracture of the lower one third of shaft of femur and decrease in bone density with irregularity of the cortices. Hyperdense area surrounded by radiolucent zone was outlined to be as sequestrum. Periosteal reaction with involucrum could also be visible on the X-ray film (Fig 1).

Diagnosis of chronic osteomyelitis on an old malunited compound fracture of distal $3^{\text {rd }}$ left femur was made. A sequestrectomy and wound debridement was planned. Under general anesthesia, a $3 \mathrm{~cm}$ incision was given over previous scar, on the medial aspect, about $5 \mathrm{~cm}$ above the left knee. Incision was deepened. Femur bone was exposed. All unhealthy tissue along with sequestrum $(4 \mathrm{~cm} X 1 \mathrm{~cm})$ was removed. Sinus tract was traced and removed. Wound was thoroughly washed with normal saline, betadine and hydrogen peroxide. Suction drain kept and wound was closed in layers. Above knee cast was applied.

Post operatively, patient was kept on antibioticsintravenous Ceftriaxone $500 \mathrm{mg}$ twice daily and Flucloxacillin $500 \mathrm{mg}$ six hourly for two weeks and switched over to oral antibiotics Cloxacillin $500 \mathrm{mg}$ six hourly for four weeks. Drain was removed after 3 days. Suture was removed after two weeks through a window created on the leg cast and the wound had healed. Patient came for follow up after one month. Repeat X-ray showed features of healing with no sequestrum. Above knee cast was removed. Patient was advised for partial weight bearing for one month and again called for follow up after one month.

Fig 1: Plain radiograph of left thigh showing features of chronic osteomyelitis in a maluntited fracture (arrow showing sequestrum).

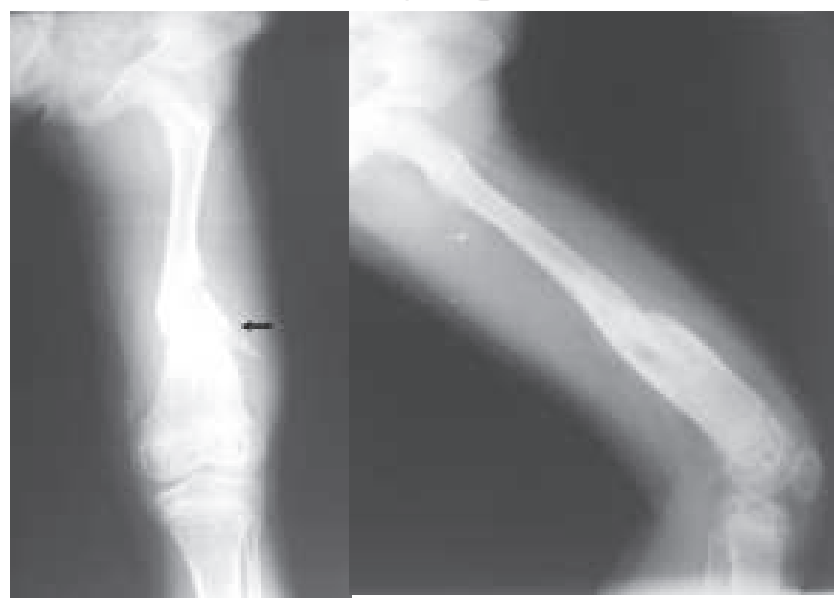

\section{Discussion}

Posttraumatic osteomyelitis following compound fracture is a common complication. Among compound fractures, it is frequent in external compounding type. ${ }^{3}$ Hematoma formed around the metaphysis of the long bones like femur, tibia, humerus permit microbial seeding after transient bacteremia. 
Journal of College of Medical Sciences-Nepal, 2012, Vol-8, No-2

The clinical manifestations of the infection get altered due to the antimicrobial agents used by the patient during the illness. So the patient may be afebrile or may just complain of limp or painful extremity. ${ }^{4}$ Our patient presented with mild pain, pus discharge and a limping gait. The course of the bone infection is affected by the local conditions at the fracture site and the virulence of the infecting organism. In the study done in Janeway Child Health Center, ${ }^{4}$ gram negative bacilli were isolated in most of the cases of secondary osteomyelitis and few were gram positive, or culture negative cases. The culture of the pus done in our patient showed no organisms.

Infection disturbs the normal union process in a fracture. ${ }^{5,6}$ The malunion in this case could be due to the fracture mal-reduction during its initial management. Thus this case holds the predisposing factor like compound fracture on a bone which is a common site for osteomyelitis.

The treatment of chronic osteomyelitis is primarily operative with support of perioperative antibiotics, rest and splintage. Copious irrigation, pulsative lavage, and antibiotic impregnated polymethyl-methacrylate (PMMA) beads ${ }^{7}$ can reduce the recurrence of infection in osteomyelitis. The surgical interventions possibly available for treating osteomyelitis secondary to compound fracture are sequestrectomy, saucerization, curettage and resection of bone ends. ${ }^{8}$ At times, after repeated failure of surgery to eradicate chronic osteomyelitis, a deformed useless, infected part becomes nuisance and may warrant amputation. Radical debridement of infected bone with bony reconstruction and soft tissue and neurovascular flap coverage are other treatment options. Despite advances in surgery and antibiotics, treating chronic osteomyelitis of the long bones is difficult; and among them, osteomyelitis of the femur is the most difficult to cure. ${ }^{8}$ In our case, sequestrectomy with debridement was performed in the distal femur and continuous suction drainage was kept. An above knee cast was applied for immobilization. Antibiotics therapy was provided for six weeks.

\section{Conclusion}

Chronic osteomyelitis is a frequent complication after a compound fracture of long bones, which is often difficult to treat. Individualization of the case, evaluation of the fracture site stability, commitment and patience from both the patient and the treating physician is essential. Aggressive thorough debridement, adequate dead space management, with bone and soft tissue reconstruction, can provide a favorable outcome. Intensive follow up of such cases for a long time is necessary.

\section{Acknowledgement}

We would like to thank the HOD and the family of Orthopedics Department, CMS-TH for their support and cooperation.

\section{References}

1. G. Cierny, J.T. Mader, H. Pennick. A clinical staging system of adult osteomyelitis. Contemp Orthop 1985; 10:17-37.

2. B. Maciuceanu, L.P. Jiga, A. Nistur, et al. Chronic Osteomyelitis of long bones. Timisoara Medical Journal 2005; 55(1): 85-100.

3. R.B. Gustilo, R.L. Merkow, D. Templeman. The management of open fractures. J Bone Joint Surg Am. 1990;72:299-304. 
A. Shrestha et al. Chronic osteomyelitis on an old compound fracture

4. R.H. Fitzgerald,D.G. Landells, J.D.E. Cowan, Osteomyelitis in children: comparison of hematogenous and secondary osteomyelitis. CMA Journal,January 25, 1975; 112: 166-9.

5. K.G. Heiple, C.H. Herndon. The pathologic physiology of nonunion. Clin Orthop 1965;43:11-21.

6. E.A. Nicoll. Fractures of the tibial shaft. A survey of 705 cases. J Bone Joint Surg (Br) 1964;46-B:373-87.
7. S.L. Henry, P.A.W. Ostermann, D. Seligson. The prophylactic use of antibiotic impregnated beads in open fractures. J Trauma 1990; 30: 1231-8.

8. Tom S Mebane. Chronic osteomyelitis secondary to compound fracture. J Bone Joint Surg Am. 1922; 4:6777. 